\title{
Development of Mechanism for Finger Prosthesis
}

\author{
Dominika Belvončíková1, Lucia Bednarčíková',**, Monika Michalíková', Branko Štefanovič', \\ Marianna Trebuňová1 , Viktória Mezencevová', Jozef Živčák ${ }^{1}$
}

Department of Biomedical Engineering and Measurement, Faculty of Mechanical Engineering, Technical University of Košice, Letná 9, 04200 Košice

Abstract: Nowadays prosthetic fingers provide not only a cosmetic solution to the problem, but mainly they provide function. 3D printing is an ideal technology for the manufacturing of low-cost prostheses, especially the upper limb prosthetics. The advantages offered by this technology make it possible to increase the level of creativity, personalization and at the same time provide space for new variants of control mechanisms regarding movement efficiency. The aim of the paper is to present variant design of a finger prosthesis manufactured by 3D printing, which have been developed and tested at the Department of Biomedical Engineering and Measurement, Faculty of Mechanical Engineering, Technical University of Košice. Those variants can flex in specified areas and copy the function of a real finger as much as possible. To ensure flexion in the joints, and at the same time achieve sufficient gripping force is a very current problem. This article offers the possibility of solving this problem, and gives evaluation of individual print variants. The results are very useful in the hand prostheses designing process and provide suitable economical solutions without reduction to the effectiveness of the prosthesis.

Keywords: 3D print; finger flexion; finger prosthesis

\section{Introduction}

Individuals with partial hand loss can be fit with either passive silicone cosmeses, passive mechanical devices, body-powered devices, or externally powered devices [1].

Body-powered devices for partial finger amputations can provide more functionality, but usually at the expense of esthetics and comfort [1]. While these devices can articulate, they require power from either a residual digit, an adjacent intact digit, or a cabling system [2]. In addition, the grip strength of body-powered devices is limited by the strength of the driving body part and the motion provided by these devices is always coupled, which limits the number of achievable grasps [3].

High end prosthetics can cost thousands of dollars, which is unfortunately not affordable for many of those in need of prosthetic hands.

The three-phalanx architecture has been the choice of many high-end hand prostheses. It can be realized through designs based on cables or linkages [4, 5]. However, this solution does not suit 3D-printed hand prostheses. Designs based on cable provide individual or coupled phalanx movements through a cable system. For 3D-printed prosthetic digits, internal joint forces are critical due to the low strength of 3D-printed components.

Zhang et al. $[4,6]$ reported concerns on instability and small grasping force for the cable designs, while pointed out the loss in grasping capacity due to high force angle. As for the alternative designs featuring one-way cable with compliant joints, fatigue of the compliant material at the joints can compromise the performance [4]. In addition, compliant joints consume additional actuation energy $[4,5]$, resulting in a reduction in the output grasping force, an increased user fatigue on the body-powered prostheses.

* Corresponding author: Lucia Bednarčíková, E-mail address: /ucia.bednarcikova@tuke.sk 
Monolithic design of prosthetic finger, which has been used in this work, reduces production costs, product weight, maintenance requirements, on the other hand, enables a comfortable, compliant and anthropometric design.

Presented prosthetic design as well as the mechanism units respected the physiological shape of finger segments. Overall, three flexion mechanisms have been designed. Flexion using a wire, lever mechanism and electromagnetic control mechanism.

An FFF type printer Tarantula and Arnitel Eco material (semiflexible material), with a tensile modulus of $250 \mathrm{MPa}$, has been used during the manufacturing process. There was no need to adjust the settings of the printer, since the material behaved similarly to PLA, therefore, default settings were selected during the printing process. Only minor adjustments were made. The printing temperature was set to $230{ }^{\circ} \mathrm{C}$ and the printing speed has been lowered to $40 \mathrm{~mm} / \mathrm{s}$.

\section{Wire flexion mechanism}

Flexion mechanism using a wire has been printed out in one piece. The first type was designed with two options depending on the shape of the finger (Fig. 1). The first one is in a physiological position ( $164^{\circ}$ in PIP and DIP) and the second one is in the shape of a finger in extension. In the first case, the prism was printed considering the angles of PIP (proximal interphalangeal) and DIP (distal interphalangeal) finger joints and it was vertically oriented so that the support leaned on the bent model parts. The gaps between digits are in the joints area and are square shaped. The model is designed as a thin-walled structure, since previous experiences determined that neither the thickness nor the infill shape affects the finger flexion to a significant degree. Inside of the model is a channel for the wire, which goes along the palmar side of the finger and ends above the fingertip on the external side.

The second mechanism option is a finger in extension. This type secures flexion with a wire mechanism, where the wire is placed within a channel, which is not ended on the distal external side of the print, but instead it curls internally in the last segment, which eliminates the risk of damaging the end part of the mechanism by external interference. Due to the channel inclusion
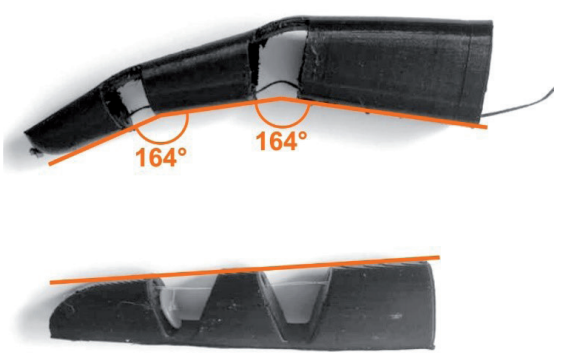

Figure 1: Both wire mechanism variants (basic position).

it is necessary for the model to be filled. The infill has a grid structure, which has proven to be the most economical solution. Another distinction from the previous model also lies in the gaps, which are in the shape of a trapezoid. Since this is a filled model and it is in extension, no supports were required to ensure stability on the plate during printing.

\subsection{Wire mechanism analysis}

The first mechanism was a construction unit imitating a finger, which uses a wire connected to the distal end of the finger that creates a moment of flexure (Fig. 2). The amount of force $F(\mathrm{~N})$ required for bending depends on the size (depth) of the gap located in the joint area and the location of the wire attachment. To simplify the calculations, the wire is perfectly rigid, so that only the finger deformation would be represented, not the deformation of the finger and wire.

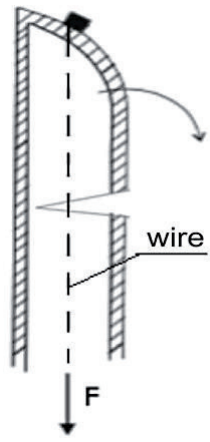

Figure 2: Wire bending mechanism, printed model in section with indicated forces.

The wire model represents the simplest solution to finger flexion from a manufacturer perspective. Fig. 3 represents an analysis model, showing the rotational (joint) connection with resistance moment $M(\mathrm{~N} . \mathrm{m})$, proportional to angle of rotation $\varphi\left(^{\circ}\right)$. The physical model contains two joint connections. From a mechanical perspective it is a 
planar mechanism with two degrees of freedom. The wire-to-model reaction is modelled by eccentrically acting force sufficient to represent reality. $L_{1}, L_{2}$ and $L_{3}(\mathrm{~m})$ are the lengths of distal, middle and proximal segments. The perpendicular distance between the point of weir attachment and the distal segment is $d(\mathrm{~m}) . A, B, C$ and $D(\mathrm{~N})$ represent reaction forces (Fig. 3).

It is necessary to define the force required to bend the finger. A kinematic condition as described by a team led by $K$. Li, MEng, PhD [7] is used for simplification between angles of rotation $\varphi_{1}$ and $\varphi_{2}$ in the form of:

$$
\varphi_{2}=\frac{3}{2} \varphi_{1}
$$

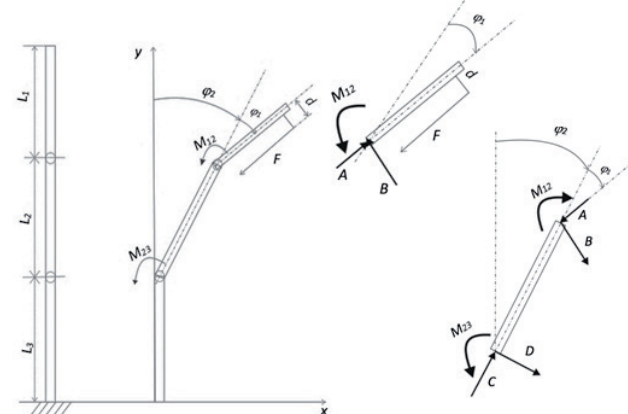

Figure 3: Left- analysis model of finger with wire mechanism, middle- relaxed distal finger segment, right- relaxed middle finger segment.

Since during finger flexion there might be no PIP joint movement, we consider this connection to be fixed. For this reason, the system has two degrees of freedom. In each the joint, reaction force A of the same size, but in the opposite direction as the external force $F$, acts to ensure balance and to prevent translational movements. Since the wearers of the two forces are displaced parallel to each other, the rotational effect of the force is counterbalanced by the moment $M_{12}$ to ensure a uniform rotation representing the bend of the finger.

$$
\begin{aligned}
& A=F \\
& B=0 \\
& M_{12}=F \cdot d
\end{aligned}
$$

The equation (4) after substituting moment $\mathrm{M}_{12}=\mathrm{k} . \varphi_{1}$ becomes:

$$
k \cdot \varphi_{1}=F \cdot d
$$

Equations for the middle segment:

$$
C=A \cdot \cos \varphi_{1}=F \cdot \cos \varphi_{1}
$$

$$
M_{12}=M_{23}+A \cdot L_{2} s \cdot \sin \varphi_{1}
$$

Substituting formulas (2) and (5) and using a similar relation for $M_{23}$ as for $M_{12}$ changes the equation (7) as follows:

$F \cdot L_{2} \cdot \sin \varphi_{1}+k \cdot \varphi_{2}=F \cdot d$

After considering kinematic condition (1) in equation (8) and adjustments, the equation for calculating magnitude of the force required to bend the finger at an angle $\varphi_{1}$ in the form of:

$$
F=\frac{3 \cdot k \cdot \varphi_{1}}{2 \cdot\left(d-L_{2} \cdot \sin \varphi_{1}\right)}
$$

Graphical imaging proved, that the finger achieves physiological bending with relatively small force.

\section{Bending mechanism - lever}

Another variant to achieve the most realistic finger flexion is with the use of a lever. The lever mechanism has been printed out in parts and a plate, connecting the levers, has been designed. In this case, three design options were made (Fig. 4), where the first ensures the bending using tensile force of a wire connected to the lever. There is a change of direction in the tensile force during manual control, and thus full range of motion is not guaranteed. This problem has been removed in the second option with the addition of a channel, which guide the wire and ensure invariability of the force $F(\mathrm{~N})$, thus securing a full range of motion. The wire of the third model that connects the outer lever and the lever glued inside leads through a small channel, which is accounted for in the actual design. The lever material used in these variants must be significantly tougher than the actual finger in order to avoid its deformation. Both sides of all levers in these designs contain an obtuse angle to ensure sufficient. The bend itself occurs at the most yielding point, that is, at the point with the least axial quadratic cross-sectional moment. This is also due to the local reinforcing effect of the rigid lever, resulting in bending at a precisely defined location, that is close to the end of connection of lever and plate, which is used in all models. 

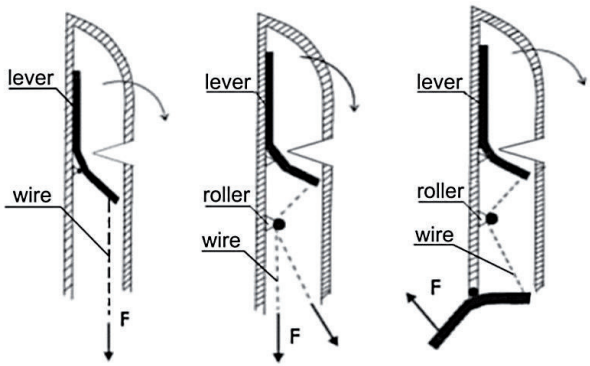

Figure 4: Three lever mechanism variants; design models in section with indicated forces.

A relaxed physical print model has been created for analysing (Fig. 5), which has been simplified while keeping all relevant model characteristics. It considers flexibility properties of the model with moment of resistance $M_{0}$ (N.m), which acts as an internal force variable in response to an external load force. Since there is no movement of the whole system in the direction of $x$ and $y$ axis, the motion equations change to static balance conditions. Since the finger movement happens at a constant angular velocity, the angular acceleration is zero.
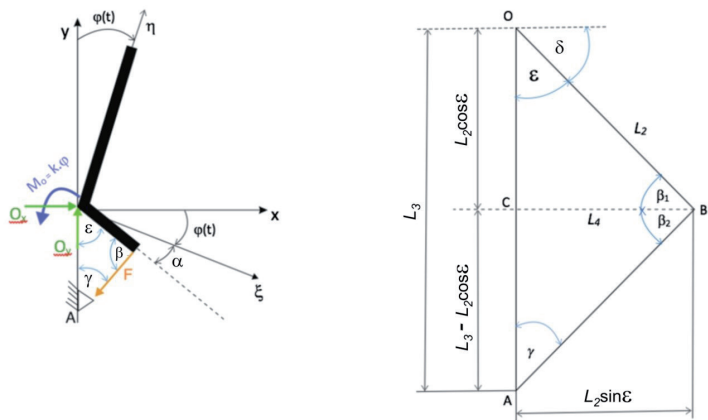

Figure 5: Physical mechanism model after relaxing.

Due to the lever toughness being several times higher than the finger material toughness, the lever deformation is ignored and is considered to be perfectly rigid. This causes transfer of the load to the finger material and flexion at the predetermined location into the desired position. Partial relaxing and investigation of the balance between external and internal force effects at the finger flexion point happen in this given case.
With the balance implementation and after adjustments it is possible to calculate the amount of force needed for finger flexion into the position set by angle $\varphi\left({ }^{\circ}\right)$ in the form of:

Based on the kinematic dependencies it is possible to determine the course of the angle of rotation $\varphi$ as a function of time. This is expressed by the basic kinematic relationship:

$\dot{\varphi}=\frac{d \varphi}{d t}=\omega$

With respect to the established coordinates and for motion at time $t_{0}=0$ is $\varphi\left(t_{0}\right)=0$, the equation (12) is adjusted as follows:

$\varphi(t)=\omega \cdot t$

By substituting equation (11) respectively (12) is possible to convert equation (10) to one of force depending on time.

\section{Flexion mechanism - electromagnet}

The last variant considers the use of an electromagnet (Fig. 6).

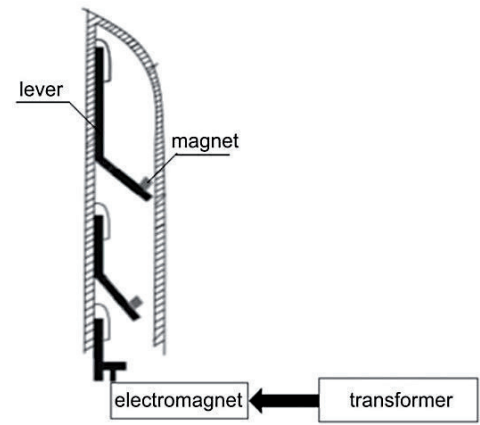

Figure 6: Flexion mechanism - electromagnet; design model in section with indicated parts.

A hollow print served as a model for the electromagnetic mechanism application. There are levers made of a ferromagnetic material placed in cases on the inside of the print. An electromagnet connected to a transformer creates a magnetic field and the increase of the magnetic fields intensity gradually draws the ferromagnetic lever of the middle segment towards the electromagnet located

$$
F=\frac{k \cdot \varphi(\mathrm{t})}{L_{2} \cdot \sin \left\{\arctan \left[\frac{1}{\tan \left(90^{\circ}-\alpha-\varphi(t)\right)}\right]+\arctan \left[\frac{L_{3}-L_{2} \cdot \cos \left(90^{\circ}-\alpha-\varphi(t)\right)}{L_{2} \cdot \sin \left(90^{\circ}-\alpha-\varphi(t)\right)}\right]\right\}}
$$


on the proximal side of the finger. After these parts connect, the lever of the middle segment becomes magnetized, which intensifies the magnetic field and transfers magnetic properties onto the next segment, creating a controlled finger flexion. For a strengthening effect of the magnetic properties there are neodymium magnets attached to the ferromagnetic parts.

\section{Discussion}

As part of the solution, 2 variants of the wire mechanism were made by 3D printing using the FFF method from Arnitel Eco material (semiflexible material).

The physiological wire mechanism model will not achieve full finger extension because of its design. The extended model differs from the physiological one in the model filling and also in the shape of gaps, thanks to which the segments manage to come closer to each other, thus leading to a greater flexion in the joins in comparison to the first printed model (Fig. 7). The measured angles show that the finger in extended basic position has greater range of motion and thus is better from a functional point of view. In comparison to the physiological model it creates greater resistance during flexion, which is due to the structure not being thin walled. A mechanical force gauge has been used to find the minimal force for bending of models to final position. The full flexion position of the physiological model in PIP joint has been achieved by $2 \mathrm{~N}$ tensile force and full flexion position in PIP and DIP has been measured $4 \mathrm{~N}$. The full flexion position of extension model in PIP joint has been achieved by 7,2N tensile force and full flexion position in PIP and DIP has been measured 9N.

Adhesion of individual layers is satisfactory and there is no need for additional printing parameter adjustments of this model. To ensure a long-lasting mechanism operation, it is necessary to consider eventual protection of the print against external factors. The wire mechanism has shown to be highly effective and fulfils its role in both printed variants.

The lever model has proven to be effective after the connection of all parts of the lever mechanism and the plate with shells. The printed finger provided flexion within physiological ranges; however, this mechanism did not achieve maximum flexion possible for a physiological finger segment (Fig. 8). The textile rubber, among other, protects
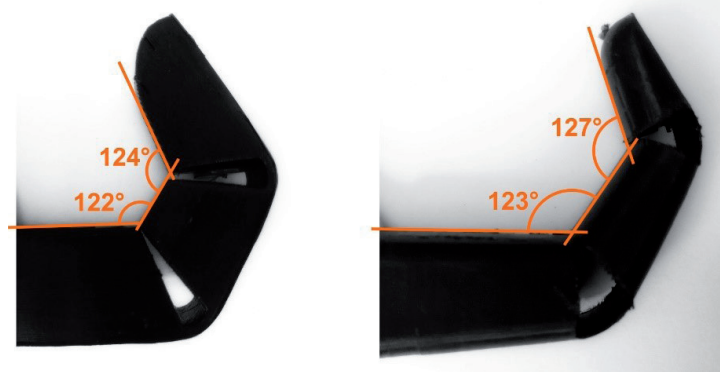

Figure 7: Physiological model in flexion (left), extension model in flexion (right).
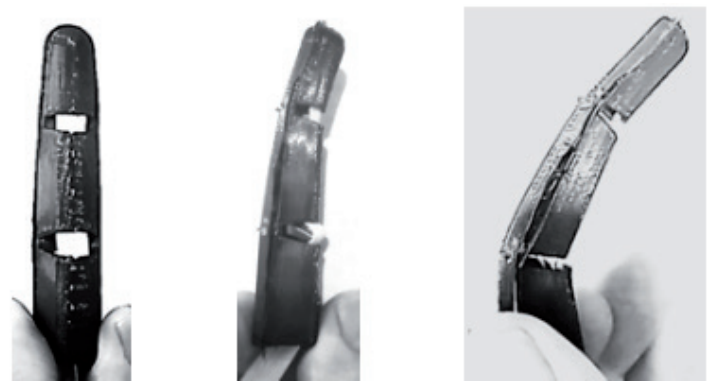

Figure 8: Print-out with lever mechanism, left - extension, right - flexion.

the print interior from external impacts. However, in comparison to the wire mechanism it is less effective.

The electromagnet connecting to the transformer (current 20V) has been constructed in two variants, in order to determine, which option is more efficient. Parallel connection, or in series, have shown to have the same level of efficiency. The designed mechanism was tested, after the testing of the electromagnet efficiency on diodes and sawdust. Ferromagnetic plates with glued on magnets have been placed through square gaps into the model. The plates were fixed on the inside with cyanoacrylate glue and slight indentations on the dorsal side of the finger. After connecting the electromagnet to the transformer in series, the desired flexion did not occur. Consequently, connection in parallel has been tested. This led to an increased finger flexion; however, the results were in comparison to the previous mechanisms unsatisfactory. Neodymium magnets have been attached on the external proximal finger side to increase the magnetic effect. However, even this change did not lead to any significant improvement of results.

This mechanism failed to provide results 
expected of the design. A solution would be the usage of plates with stronger ferromagnetic properties or a stronger transformer.

\section{Conclusions}

Testing has proven that the wire flexion mechanism is the most effective mechanism. Both variants demonstrated the greatest degree of flexion in finger joints among all testing models. The mechanism using electromagnet was not effective as expected. For future application of this type of finger flexion, it is recommended that certain changes in the connection mechanism should be applied. The chosen metal plates should be of a material with higher degree of magnetic properties or should be connected to a stronger transformer. Alternatively, it is recommended to use miniature parts so that the electromagnet can be applied directly into the printed model.

\section{Acknowledgments}

\section{This work has been produced with support under the projects KEGA 041TUKE-4/2019, KEGA 023TUKE-4/2020, VEGA 1/0316/18 and APVV-19-0290.}

\section{References and Notes}

1. Imbinto I, Peccia C, Controzzi M, Cutti AG, Davalli A, Sacchetti R, Cipriani C. Treatment of the Partial Hand Amputation: An Engineering Perspective. IEEE Rev Biomed Eng. 2016;9:32-48. doi: 10.1109/RBME.2016.2523799. Epub 2016 Jan 29. Erratum in: IEEE Rev Biomed Eng. 2018;11:322. PMID: 26849872

2. Uellendahl, JE, Uellendahl, EN.: Experience fitting partial hand prostheses with externally powered fingers. In: Vaillancourt, C, Lafond, J (eds) Grasping the future: advances in powered upper limb prosthetics. Sharjah, United Arab Emirates: Bentham Science Publishers, 2012, pp. 15-27.

3. Stevens, P.: The expanding options of partial hand prostheses. Orthot Prosthet Edge 2015; 14 (4), https:// opedge.com/Articles/ViewArticle/2015-04_03?mf=1

4. Liu S., Van M., Chen Z., Angeles J., Chen C.: A novel prosthetic finger design with high load-carrying capacity, Mechanism and Machine Theory, Volume 156, 2021 104121, ISSN 0094-114X, https://doi.org/10.1016/j. mechmachtheory.2020.104121.

5. Ceccarelli, M., Zottola, M.: Design and simulation of an underactuated finger mechanism for LARM hand, Robotica, 35 (3) (2017), pp. 483-497

6. Zhang T, Wang XQ, Jiang $L$, Wu X, Feng W, Zhou D, et al. Biomechatronic design and control of an anthropomorphic artificial hand for prosthetic applications. Robotica. Cambridge University Press; 2016;34(10):2291-308.

7. Li, K., Chen, IM., Yeo, SH., Lim, CK. 2011. Development of finger-motion capturing device based on optical linear encoder. J Rehabil Res Dev. 48(1):69-82. doi:10.1682/ jrrd.2010.02.0013

8. Baich, L.J., Manogharan, G., \& Marie, H. 2016. Study of infill print design on production cost-time of 3D printed ABS parts. International Journal of Rapid Manufacturing. BQ Store. Filaflex Skin2 1,75mm 500 gram. BQ Store Nederland [online]. Arnhem (Netherlands).

9. Jensen, K.F. 2018. Exploring Manufacturing Options for Elastomeric Components. ProtoLabs [online].

10. Rayna, Thierry \& Striukova, Ludmila. 2014. The Impact of 3D Printing Technologies on Business Model Innovation. 10.1007/978-3-319-04313-5_11.

11. Živčák, J., Hudák, R., Tóth, T., Rajtúková, V., Michalíková, M., Majerník, J., Frankovský, P. 2018 Biomechanika človeka I., Prešov: Grafotlač Prešov, ISBN 978-80-553-3221-5

12. Resinex. ARNITEL ${ }^{\oplus}$ TPE-E VLASTNOSTI. Resinex [online]. Prague, 2019

13. Cabibihan, J.J., Abubasha, M.K., Thakor, N. 2018. A method for 3-D printing patient-specific prosthetic arms with high accuracy shape and size, IEEE Access, 6 (2018), pp. 2502925039

14. Alkhatib, F, Cabibihan, J-J, Mahdi, E. 2019. Data for benchmarking low-cost, 3D printed prosthetic hands. Data Br [Internet]. 2019;25:104163.

15. Alvial, P, Bravo, G, Bustos, MP, Moreno, G, Alfaro, R, Cancino, $R$, et al. 2018. Quantitative functional evaluation of a 3Dprinted silicone-embedded prosthesis for partial hand amputation: A case report. J Hand Ther .2018;31(1):129-36.

16. Carbone G, Rossi C, Savino S. Performance Comparison Between FEDERICA Hand and LARM Hand. International Journal of Advanced Robotic Systems. July 2015. doi:10.5772/60523

17. Moon, Y.M. 2007. Bio-mimetic design of finger mechanism with contact aided compliant mechanism. Mechanism and Machine Theory, Volume 42, Issue 5, 2007, Pages 600-611. ISSN 0094-114X, https://doi.org/10.1016/j. mechmachtheory.2006.04.014. 